\title{
A Personalized Recommendation Model Based on Users' Information Behavior
}

\author{
Suming $\mathrm{Li}^{1, \mathrm{a}}$, Xianqin $\mathrm{Li}^{1, \mathrm{~b}}$ \\ ${ }^{1}$ School of National Education, Nanchang Institute of science \& Technology, Nanchang, 330108, \\ China; \\ aniefengzju@163.com, 'blunwenzju@163.com
}

Keywords: Network Users, Information Behavior, Personalized Recommendation.

\begin{abstract}
By collecting and analyzing the users' information behavior, the users' real interest can be obtained, and the recommendation of users' need can be generated. This paper explores the basic concept and the related technology of personalized recommendation. On that basis, this paper proposes a personalized recommendation model based on users' information behavior. The experiment shows that this model has some advantages in the accuracy, the stability and the efficiency of recommendation.
\end{abstract}

\section{Introduction}

There is an increasing variety of information in modern life. And the generated speed of information has multiplied at astonishing rate. At the same time, the information quality varies widely. The yield spam is more than that of the valued information. It makes the much of valued information cove the valued information. So people are difficult to find the necessary information related to them [1,2]. The personalized recommendation technology can actively recommend the needed information by analyzing the users' information behavior in real time, so that people can effectively solve the difficult of acquiring the information. The tradition personalized recommendation is based on the similarity of the users or goods. The recommendation technology is based on the past interest of users, and can hardly show the present interest of users. Based on the personalized recommendation of users' information acquires the needed interest now by using the related technology and analyzing users' information behavior, so as to generate the needed recommendation of users. This paper explores the basic concept and the related technology of the personalized recommendation, and proposes the personalized recommendation model based on the users' information behavior in accordance with that. By the compared experiments, we find that the recommendation model has some advantages in the accuracy, the stability and the efficiency of recommendation.

\section{The Basic Concept of the Users Information Behavior}

The so-called the network users are the users of acquiring and exchanging the information by network. From this, the network users can use the network information, namely, can operate the network information in accordance with own needed network information. In addition, the network users can also create the network information, namely, the network users can also create network information when they operate the new network information [3]. Some experts think the network users' information behavior is a rational behavior with meeting the information needs. There behaviors include a range of processing, such as querying information, gathering information, dealing with information, generating information, using information and spreading information. But I think that the network users' information behavior is referred that the network users implement some activities by using the network, such as information query, information exchange, information selection, information absorption and information utilization, etc [4]. According to the process of behavior activity and the type of behavior activity, the network users' information behavior can be classified into information requirement perception \& expressive behaviors, information query 
behavior, information interaction behavior, information selection behavior and information absorption \& utilization behavior [5].

The generation and formation of the network users' information behavior is not incidental, but it is result of interaction of user principal and its environment, so it will be influenced by the users themselves and environmental factors. Generally speaking, the influence factors of network users' information behavior mainly include individual factor, information \& environment factor, society \& natural factor, etc.

\section{The Related Technology of Personalized Recommendation}

The personalized recommendation refers to implement some operations (such as browse, purchase, evaluation and search, etc) by analyzing network users, so as to filter information that may be useful to the users, and actively recommend the information to the network users in some way. At present, the personalized recommendation technology includes recommendation based on association rule, recommendation based on content filtering and recommendation based on collaborative Filtering.

The association rule can reflect the association and dependence of two or more things. If there are some associations among two or more things, then we can predict other things though one of the things.

The recommendation based on content filtering also calls the recommendation based on item and item correlation. It can recommend the old similar-item to users in accordance with history. The recommendation technology is built upon the similar association rule mode of item-to-item. This rule is determined by relations of the purchase data, co-visited data and content similarity, namely, if item $A$ is purchased, then item $B$ will be purchased. In the recommendation system, if item $A$ is highly interrelated to item $B$, the item $B$ will be recommended to the present users when the present user purchases the item $A$. The recommendation principle is shown in Figure 1.

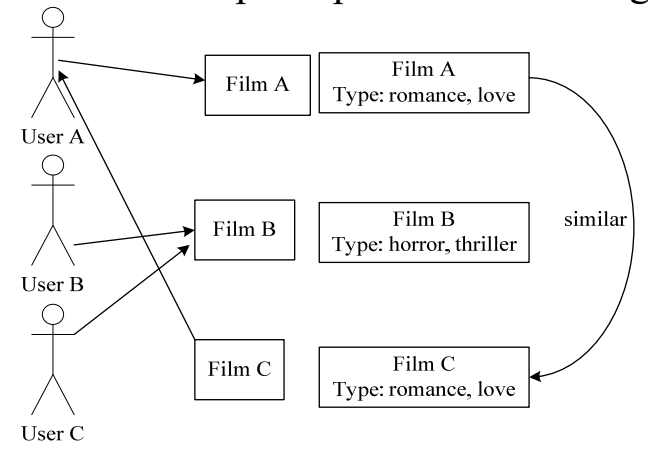

Fig.1 Recommendation Principle Content-based Filtering

The collaborative filtering recommendation calls is also called recommendation based on user-to user correlation. It is based on opinions of other users, and can discover interesting content of new users. The collaborative filtering recommendation recognizes the neighbors of user in accordance with history information, and recognizes the favorite information of user by analyzing these neighbors of users. Unlike the content-based recommendation, the collaborative filtering recommendation is not based on similar item that users once enjoyed, but based on other users' preferences. It doesn't need calculate the similarity of items, only need calculate the similarity of users. The collaborative filtering recommendation includes mainly collaborative filtering recommend based on user and collaborative filtering recommend based on item.

These recommendation methods have their advantages and disadvantages. In ordered to acquire better the recommendation outcome, some hybrid recommendations are often used on recommendation system. The hybrid recommendation is not simply a combination of multiple recommendation methods. More important, the hybrid recommendation can avoid or make up the disadvantages of recommendation technology. In the hybrid way, some researchers propose seven hybrid ideas, such as weigh, switch, mixed, feature combination, cascade, feature augmentation and meta-level, etc. 


\section{Personalized Recommendation Model Based on Users' information Behavior}

The traditional personalized recommendation is a recommendation technology based on the similarity of items or users. Owing to the traditional personalized recommendation recommend information to users by old interests of users, and it cannot reflect the present interests of users because of the present interests of users. The recommendation system cannot recommend items that users really need. Compared with the traditional personalized recommendation, the recommendation technology based on users' information behavior acquire real interest by using some related recommendation and analyzing the users' behaviors, and produce recommendation that users really need. That way, it can save the time of information retrieval, and improve the accuracy. The recommendation model is shown in Figure 2.

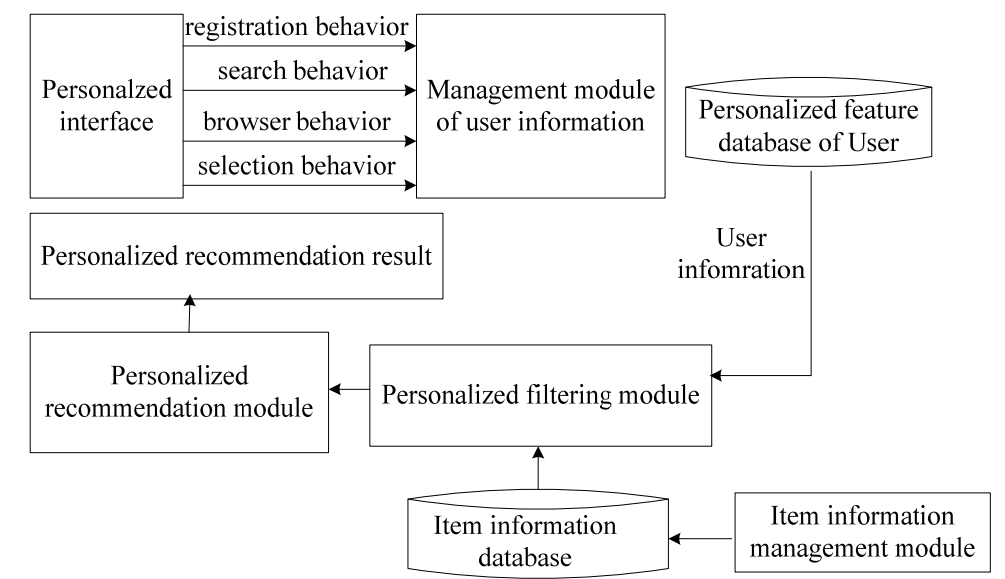

Fig.2. Personalized Recommendation Based on Users' Information Behavior

The recommendation model includes four modules, such as: item information management module, user information management module, personalized filtering module and personalized recommendation module.

1) Item Information Management Module. It is responsible for management of network item data information and recommendation item data.

2) User Information Management Module. Some information of interest user browse, search and purchase item was stored into the related database. It can predict personal preferences of user in accordance with history retrieve, browse and spending records of users.

3) Personalized Filtering Module. According to users' information model management module, the personal preferences of user can be acquired. And compare the item data you can acquire item information of personal preferences of user.

4) Personalized Recommendation Module. The recommendation module can automatically recommend automatically the item information to user.

Some experts and scholars of Chinese and oversea compared these recommendation methods by basic data online. Zhang[6] contrasted the three recommendation methods by using MovieLens. MovieLens includes some information, such as user information, movie information, and user rating, etc. The user rating represents preference that the network users recommend for personalized services.

The personalized recommendation model based on users' information behavior can get users' preference by filtering some operation (register, retrieval, browse and selection) of related users, and recommend information to user in accordance with user preference. The other two recommendation models were based on similarity between items and items or users and users. In order to compare three recommendation models, the reference 6 implements related experiment by selecting the three indexes of mean absolute error ( hereafter referred to as MAE), standard variance of mean absolute error (hereafter referred to as SV.MAE) and average prediction time ( hereafter referred to as APT). The researcher selected randomly a percentage of data as the training set in the experiment, and the rest were taken as test dataset. The result of two indexes was calculated by repeated experiments. The result is shown in Table 1. 
Table1. Comparison of Three Recommend Models

\begin{tabular}{ccccc}
\hline Recommendation Model & Given & MAE & SV.MAE & APT \\
\hline \multirow{2}{*}{ Personalized recommend model based } & 5 & 0.71 & 0.55 & 26.2 \\
on users ' information behavior & 21 & 0.70 & 0.52 & 24.8 \\
& All But 1 & 0.70 & 0.50 & 25.4 \\
Recommendation model based on & 5 & 0.94 & 0.72 & 30.9 \\
collaborative filtering & 20 & 0.90 & 0.68 & 29.4 \\
& All But 1 & 0.80 & 0.57 & 27.2 \\
Recommendation model based on & 5 & 0.88 & 0.71 & 89.4 \\
content & 20 & 0.84 & 0.68 & 84.5 \\
& All But 1 & 0.76 & 0.64 & 80.7 \\
\hline
\end{tabular}

Table1 shows that the recommendation model based on users' information behavior is better than the other two models in MAE. In this regard, the recommendation model based on users' information behavior can better deal with the data sparse problem, and improve the recommendation effect of the recommendation model. From the view of APT, the recommendation model based on users' information behavior is less than the other two recommendation model in average time. In this regard, the recommendation model based on users' information behavior has some advantages of short recommendation time and high recommendation efficiency.

\section{Summary}

The tradition personalized recommendation is based on the similarity of the users or goods. The recommendation technology is based on the past interest of users, and can hardly show the present interest of users. The personalized recommendation based on of users' information behavior acquires the needed interest now by using the related technology and analyzing users' information behavior, so as to generate the needed recommendation of users. The related experiment indicates that the personalized recommendation based on of users' information behavior has some advantages in the accuracy, the stability and the efficiency of recommendation.

\section{References}

[1]. A. L.DENG, Y.Y. ZHU, B.L. SHI. The Collaborative Filtering Algorithm Based on Prediction of Item Rating, Journal of Software, 14(2003),p.1621-1625.

[2]. D.W.PENG, B.HU. A Collaborative Filtering Recommendation Algorithm Based on Users Feature and Time, Wuhan Technology University Journal, 2(2009), No.18. p. 24-28.

[3]. Y.H, SHAO. Research Situation and Development Tendency of the Users' information Behavior, Information Exploration. 2(2011), No.11.p. 18-20.

[4]. L. XU, X.WU, X.D. LI. Comparison Study of Internet Recommendation System. Journal of Software, 14(2003) ,No.2. p.350-362.

[5]. Q.WANG, J.B.WANG. An Improved the Collaborative Filtering Recommendation Algorithm, Computer Science, 6 (2010), p. 226-228.

[6]. L.L.ZHANG. Research on E-commerce Personalized Recommendation Model. Chongqing University, china.2008. p. 125-132. 